\title{
Resonant beam behavior studies in the Proton Storage Ring
}

\author{
S. Cousineau, J. Holmes, and J. Galambos \\ Oak Ridge National Laboratory, Oak Ridge, Tennessee, USA \\ A. Fedotov and J. Wei \\ Brookhaven National Laboratory, Upton, New York, USA \\ R. Macek \\ Los Alamos National Laboratory, Los Alamos, New Mexico, USA
}

(Received 12 February 2003; published 31 July 2003)

\begin{abstract}
We present studies of space-charge-induced beam profile broadening at high intensities in the Proton Storage Ring (PSR) at Los Alamos National Laboratory. We investigate the profile broadening through detailed particle-in-cell simulations of several experiments and obtain results in good agreement with the measurements. We interpret these results within the framework of coherent resonance theory. With increasing intensity, our simulations show strong evidence for the presence of a quadrupole-mode resonance of the beam envelope with the lattice in the vertical plane. Specifically, we observe incoherent tunes crossing integer values, and large amplitude, nearly periodic envelope oscillations. At the highest operating intensities, we observe a continuing relaxation of the beam through space charge forces leading to emittance growth. The increase of emittance commences when the beam parameters encounter an envelope stop band. Once the stop band is reached, the emittance growth balances the intensity increase to maintain the beam near the stop band edge. Additionally, we investigate the potential benefit of a stop band correction to the high intensity PSR beam.
\end{abstract}

DOI: 10.1103/PhysRevSTAB.6.074202

PACS numbers: 29.27.Bd

\section{INTRODUCTION}

Future high intensity synchrotrons will require unprecedented control of beam loss in order to comply with radiation safety regulations and to allow for safe, handson maintenance of machine hardware. A major cause of beam loss in high intensity synchrotrons is the space charge force of the beam, which can lead to beam halo and emittance dilution. A better ability to understand, predict, and mitigate space-charge-related effects is necessary to reach and exceed the design goals of these machines.

The quantity of merit in assessing the effects of space charge has traditionally been the incoherent space charge tune shift; machine working points are typically chosen to avoid low-order resonances, particularly the nearest underlying integer or half-integer value. Recently, it has been recognized that the coherent motion of the beam is more relevant in describing the resonant behavior. In his doctoral thesis, Sacherer used the particle core framework developed by Kapchinskij and Vladimirskij [1] to perform complete analyses of the periodic coherent halfinteger envelope resonance [2]. His work demonstrated that the incoherent space charge tune shift alone is an insufficient measure of the resonance threshold, and that the onset of the space-charge-induced resonance occurs at tune shifts in excess of the incoherent stop band limit. At these tune shifts, his model predicts that the beam envelope undergoes periodic oscillations about the zerospace-charge envelope, and that the amplitude of these oscillations increases nonlinearly with the level of space charge in the beam.

The qualitative nature of Sacherer's findings has been confirmed [3-6], and the analysis of the coherent envelope resonance has been extended to describe arbitraryorder periodic resonances for two-dimensional beams $[7,8]$. More recent work has extended into the experimental realm, where measurements of the coherent tune of the beam have been accomplished [9]. At this time, it remains to strengthen the connection between theory and experiment and to validate the predictions of models used in the design of next generation high intensity rings.

The Proton Storage Ring (PSR) at Los Alamos National Laboratory is the accumulator ring portion of the Los Alamos Neutron Science Center (LANSCE) accelerator [10]. The $90.26 \mathrm{~m}, 800 \mathrm{MeV}$ synchrotron can operate with substantial space charge effects, and therefore provides an ideal laboratory for experimental studies. At the highest operating intensities, the PSR beam consistently experiences beam profile broadening in the vertical plane, accompanied by an escalation of losses that ultimately limits the attainable intensity of the machine. In an earlier study [11], the space charge dynamics of a high intensity synchrotron beam were explored through a particle core model approach that included the effects of lattice structure and finite momentum spread. Previous work with PSR data demonstrated successful benchmark of a number of experimental beam profiles [12] using the ORBIT particle-in-cell (PIC) tracking code $[13,14]$. The 
beam profile broadening at high intensity was observed in these studies, but no attempt was made to explore the physics behind the broadening; such an attempt was previously begun but not completed [4]. In this document, we investigate the behavior of the high intensity PSR beam systematically for several scenarios through detailed simulations of experimental measurements. The ORBIT PIC code is used as a tool for probing the full parameter space of the PSR beam during the accumulation cycle, and the results are combined with an envelope model to establish a comprehensive understanding of the space charge dynamics. We explore the coherent half-integer envelope resonant excitation and observe the presence of a stop band in the envelope phase space of the beam. We demonstrate that once the beam nears the edge of the stop band, space-charge-induced emittance growth balances the intensity increase to maintain fixed envelope dynamics.

The paper is organized as follows: In Sec. II we present a typical high intensity PSR beam accumulation scenario, and describe the ORBIT PIC tracking code simulations. In Sec. III, the parameter space of the high intensity PSR beam is examined through PIC simulations and calculations of the envelope equations of motion, and the envelope stop band is discussed. In Sec. IV, three experiments that measure the sensitivity of the beam to the stop band are presented. In Sec. V, the possibility of stop band correction is explored through the use of an idealized PSR lattice structure. Finally, conclusions are given in Sec. VI.

\section{PSR EXPERIMENTS AND SIMULATION}

The LANSCE facility accelerates $\mathrm{H}^{-}$ions through a linac to an energy of $800 \mathrm{MeV}$, after which they are stripfoil injected as protons into a $90.26 \mathrm{~m}$ circumference accumulator ring for bunch train compression and extracted into a transfer line for neutron spallation. For typical operating conditions, the PSR accumulates approximately $3.5 \times 10^{13}$ protons per pulse in about $675 \mu \mathrm{s}$, with a repetition rate of $20 \mathrm{~Hz}$. For PSR development or beam physics experiments, the beam can be accumulated for up to $1225 \mu$ s with a few $\mathrm{Hz}$ repetition rate and typical beam intensities of about $5 \times 10^{13}$ protons. The beam intensity can be varied by chopping the beam at the source such that the beam is accumulated on every turn for the full intensity, every other turn for onehalf of the full intensity, or every fourth turn for onequarter of the full intensity; the number of total turns remains unchanged. In the ring, vertical beam painting is achieved by linearly decreasing the vertical closed orbit bump during the injection cycle. The horizontal closed orbit remains fixed throughout the accumulation, but beam spreading occurs through nonzero dispersion at the injection point. A single harmonic, rampable rf cavity is used to maintain the beam gap, and at the end of the accumulation cycle a single-turn beam extraction is performed. Beam profiles are subsequently measured on wire scanners located in the transport channel to the neutron target. Longitudinal profiles are recorded on a wall current monitor in the ring.

Table I shows the parameters for a high intensity beam accumulation experiment. Here, $4.37 \times 10^{13}$ protons are accumulated in $1.16 \mathrm{~ms}$, i.e., $1.36 \times 10^{10}$ protons are injected on every turn for 3214 turns. Bare lattice tunes are set to $\left(\nu_{x}, \nu_{y}\right)=(3.19,2.19)$, which will be the case throughout this document, unless otherwise specified. Also shown in the table are the linac beam Twiss parameters at the injection foil, $\beta_{\text {linac }}$ and $\alpha_{\text {linac }}$, the rms emittances of the bi-Gaussian linac beam emittance, $\epsilon_{\text {linac,rms }}$, and the linac beam spot position and closed orbit bump coordinates. It should be noted that the values in Table I are not the baseline values for the PSR machine.

The ORBIT PIC tracking code is used to simulate all of the experiments. The code is designed to handle detailed

TABLE I. PSR parameters.

\begin{tabular}{lc}
\hline \hline PSR ring circumference & $90.26 \mathrm{~m}$ \\
Kinetic energy & $800 \mathrm{MeV}$ \\
Full beam intensity & $4.37 \times 10^{13}$ protons \\
Injection period & $1.16 \mathrm{~ms}$ \\
Linac beam horizontal offset & $x=2.62 \mathrm{~mm} ; x^{\prime}=-0.71 \mathrm{mrad}$ \\
Linac beam vertical offset & $y=14.00 \mathrm{~mm} ; y^{\prime}=2.28 \mathrm{mrad}$ \\
Vertical closed orbit bump at foil & $9.37 \rightarrow 0 \mathrm{~mm}$, and $1.37 \rightarrow 0 \mathrm{mrad}$ \\
Linac horizontal Twiss parameters & $\beta_{\text {linac }, x}=1.25 \mathrm{~m} ; \alpha_{\text {linac }, x}=0$ \\
Linac vertical Twiss parameters & $\beta_{\text {linac, } y}=3.20 \mathrm{~m} ; \alpha_{\text {linac, } y}=0$ \\
Linac horizontal distribution & $30 \%$ with $\epsilon_{\text {linac,rms }}=0.165 \pi \mathrm{mm} \mathrm{mrad}$ \\
& $70 \%$ with $\epsilon_{\text {linac,rms }}=0.05 \pi \mathrm{mm}$ mrad \\
Linac vertical distribution & $40 \%$ with $\epsilon_{\text {linac,rms }}=0.165 \pi \mathrm{mm} \mathrm{mrad}$ \\
Bare lattice tunes & $60 \%$ with $\epsilon_{\text {linac,rms }}=0.05 \pi \mathrm{mm}$ mrad \\
rf voltage & $\left(\nu_{x}, \nu_{y}\right)=(3.19,2.19)$ \\
\hline \hline & $16.917 \mathrm{kV}$, nonramped \\
\hline
\end{tabular}


transport of beams in rings, and uses MAD files to create the accelerator lattice. The code features sophisticated injection painting capabilities, rf cavity models, space charge and impedance modeling, and a complete set of diagnostics. The details of the code are given in Ref. [13], and a good description of the space charge implementation is given in Refs. [12,14]. In the present simulations, a number of effects are ignored, including strip-foil scattering, magnet errors, chromatic effects, transverse impedances, and higher order magnetic fields. Therefore, space charge and longitudinal impedance provide the only nonlinearities.

For all of the simulations performed, 50 macroparticles were injected on every turn, resulting in approximately $1.6 \times 10^{5}$ macroparticles by the end of accumulation. A fast-Fourier transform (FFT) method on a $64 \times 64$ rectangular grid was used to calculate the nonlinear transverse space charge of the beam [14]. Transverse space charge kicks numbering 371 were applied per turn of the beam, yielding an average integration step size of $24 \mathrm{~cm}$. The step size, grid size, and number of macroparticles have been optimized for numerical convergence [15]. Because the synchrotron motion in the PSR beam is very slow, the longitudinal space charge is represented by a single space charge kick per turn. The beam is distributed longitudinally over 64 bins, and a combined space charge and impedance algorithm adopted from MacLachlan [16] is applied.

Figure 1 shows an example of a benchmark for the experiment presented in Table I. The experimental vertical beam profile recorded on the wire scanner is shown along with the simulated beam profile at the same point in the extraction channel. The centers of the profiles were aligned, and both profiles were normalized to the same total beam intensity; neither the shape nor the width of

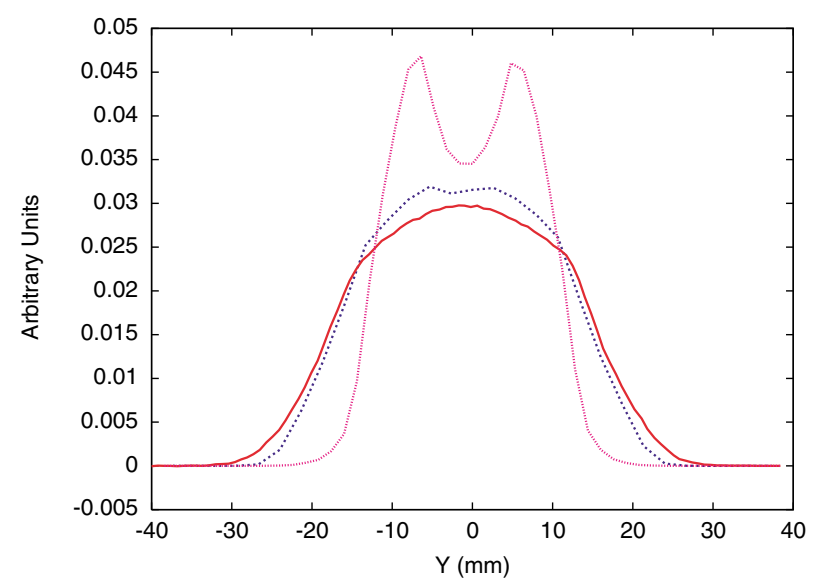

FIG. 1. (Color) Benchmark of vertical experimental profile measurements with PIC simulations. The solid (red) curve is the experimental profile. The dotted (blue) curve is the PIC result with space charge. The (pink) dashed line is the PIC result without space charge. either profile was altered. The agreement between the experimental and simulated curves is very good, with about 3\% difference in the width of the beams. Figure 1 also includes, for comparison, a simulated profile generated without space charge. The profile shape for this latter case is determined exclusively by the painting scheme employed, and a large difference from the experimental measurement is evident. Including the space charge interactions of the beam is therefore critical for accurate modeling of the experiment.

Figure 2 shows the longitudinal benchmark for the same experiment, where again the centroids of the profiles have been aligned and the vertical axes normalized to the integrated beam intensity. An inductive insert in the PSR lattice is modeled using a longitudinal impedance algorithm, implemented in conjunction with the longitudinal space charge routine. The profile simulated with the complete longitudinal package (space charge and impedance) is in close agreement with the experimental profile. Also shown in the figure is a simulated profile generated without the impedance of the inductive insert. The profile underestimates the amount of central peaking in the experimental measurement. Thus it is important to include the relevant longitudinal nonlinearities (impedance and space charge) in the simulation.

The success of these benchmarks lends credibility to the ORBIT code as a tool that can accurately portray the relevant physics in the high intensity PSR beam. The results of ORBIT simulations will now be used extensively to probe the parameter space of the beam not available through experimental measurement. We hereafter refrain from presenting benchmarks of the simulations, as this topic is covered quite thoroughly in Ref. [12]; however, it should be noted that for all experiments discussed, the

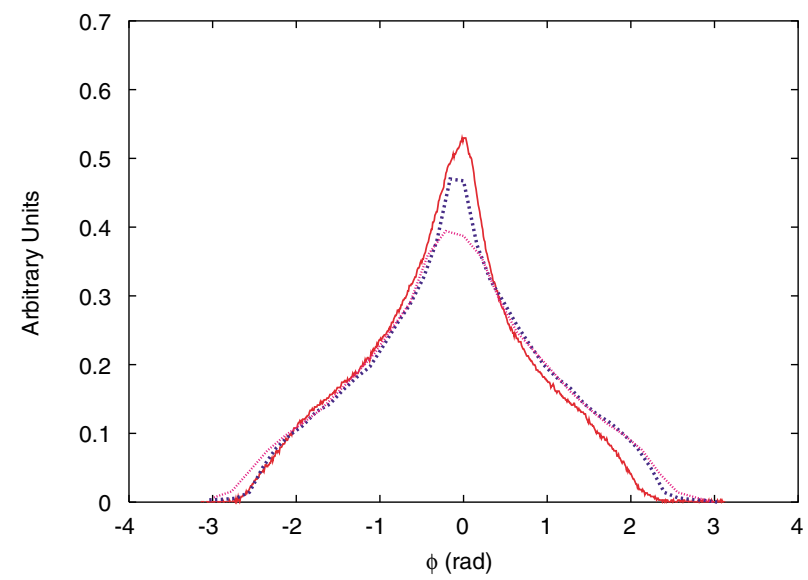

FIG. 2. (Color) Benchmark of longitudinal profile measurements with PIC simulations. The solid (red) curve is the experimental profile. The dashed (blue) curve is the PIC result with longitudinal space charge and impedance. The (pink) dotted line is the PIC result with longitudinal space charge but without other impedance. 
corresponding simulations have met successful benchmark criteria, similar to Figs. 1 and 2.

\section{PSR BEAM BROADENING AT HIGH INTENSITIES}

For typical injection scenarios, the PSR vertical beam profile undergoes significant broadening at intensities $\gtrsim$ $3 \times 10^{13}$. An example of the phenomenon is shown in Fig. 3, where experimentally measured vertical beam profiles are shown for the parameters in Table I, at three different beam intensities: the full intensity $\left(4.37 \times 10^{13}\right.$ protons), half of the full intensity, and one-quarter of the full intensity. The two lower intensity cases are nearly identical and preserve the beam distribution inherited from the painting scheme. A small hollow is seen in the center of these profiles resulting from the $4.63 \mathrm{~mm}$ difference between the initial closed orbit bump and the linac beam offset. Despite the doubling of the space charge force between the one-fourth and the one-half intensity cases, there is no obvious consequence to the beam. At the highest intensity, however, significant beam broadening occurs, and the central hole is replaced by a wide peak. Experimentally, above the threshold of observable broadening, the profile width grows with increasing intensity, until, finally, the escalating beam losses reach a maximum limit for safe operation.

PIC simulations of each of the three intensities shown in Fig. 3 have been performed, and calculated profiles are in good agreement with the experimental data. The observed experimental profile broadening is reflected in the simulations through emittance growth of the beam. Figure 4 shows the vertical rms beam emittance generated via simulation during the accumulation of the full, one-half, and one-fourth intensity beams. The slow emit-

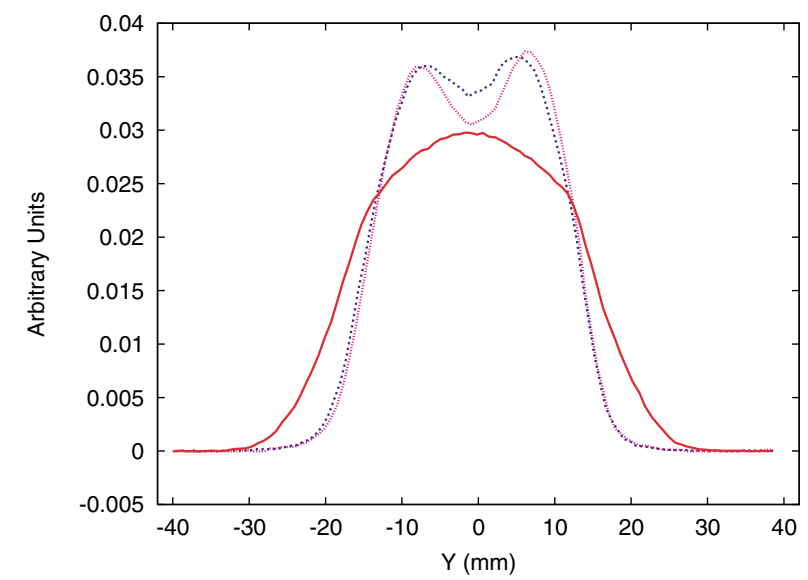

FIG. 3. (Color) Experimental vertical beam profiles after the accumulation of the full intensity PSR beam (red solid curve), the half intensity beam (blue dotted curve), and the quarter intensity beam (pink dashed curve). The three profiles have been normalized to give identical areas under the curves. tance growth for the one-quarter and the one-half intensity beams is due exclusively to injection painting effects. At the highest intensity, the emittance grows much faster after the first 1000 injection turns, i.e., when about $1.35 \times$ $10^{13}$ protons are in the ring. This is nearly the same beam intensity as the quarter intensity beam at the end of accumulation, but the difference here is that the $1.35 \times$ $10^{13}$ protons are painted into a much smaller emittance, and thus they experience considerably stronger space charge forces. Although it is clear from both simulation and experiment that space charge influences the behavior of the beam at high intensity, further exploration of the beam parameter space is necessary to provide a comprehensive understanding of the beam response to the space charge, including the onset of emittance growth.

It is insightful to calculate the vertical tune footprint of the beam at each of the three intensities considered. The simulated vertical incoherent tune shifts versus the longitudinal coordinate of the beam are plotted in Fig. 5, along with a horizontal line marking the incoherent integer stop band $\left(\nu_{y}=2.0\right)$. The large tune depression visible in the center of the bunch is due to the centrally peaked longitudinal beam density (see Fig. 2). The quarter intensity beam is entirely above $\nu_{y}=2.0$, and the half intensity just reaches this value at the bunch center. Incoherent resonance theory predicts an increase in beam emittance as tunes approach the incoherent integer stop band, followed by substantial beam loss in crossing the threshold. In contrast, recall from Fig. 3 that no beam broadening is observed in the half intensity beam, implying that the incoherent tunes can reach integer values without repercussion. This result is in agreement with the predictions of Sacherer's model. At the full intensity, where the beam broadening is observed, the incoherent tunes are depressed well beyond $\nu_{y}=2.0$, with particles at the very center of the bunch reaching $\nu_{y} \cong 1.9$.

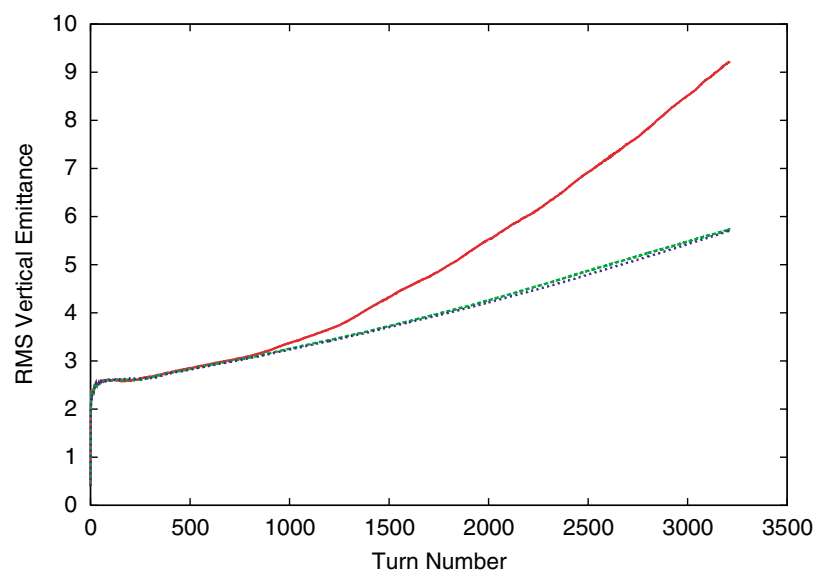

FIG. 4. (Color) Vertical rms emittance (in units of $\pi \mathrm{mm} \mathrm{mrad}$ ) evolution of the full intensity beam (solid red curve), the half intensity beam (dashed green line), and the quarter intensity beam (dotted blue line), obtained from PIC simulations. 


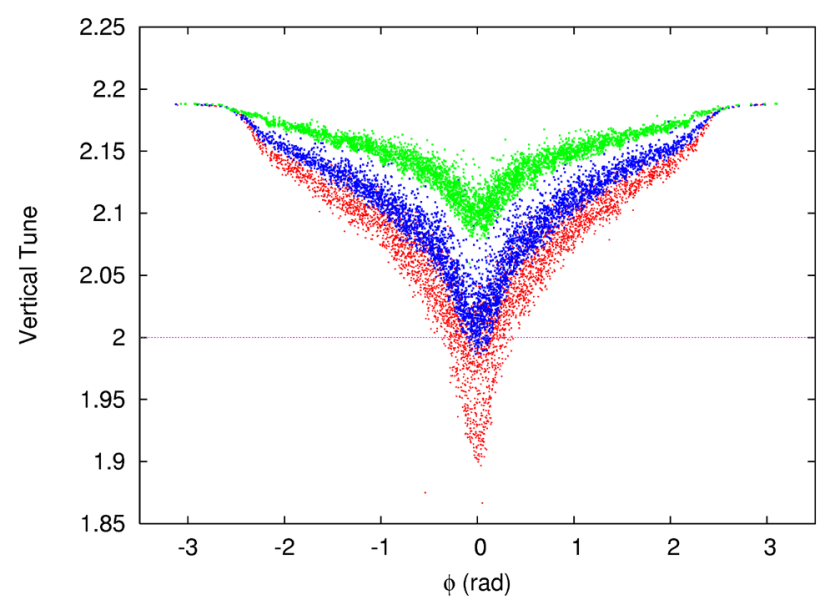

FIG. 5. (Color) Incoherent particle tune shifts versus longitudinal coordinate after the accumulation of the full intensity PSR beam (red points), the half intensity beam (blue points), and the quarter intensity beam (green points), obtained from PIC simulations.

In addition to single particle tunes crossing the incoherent stop band limit, the behavior of the beam envelope deviates from the zero-space-charge case, as predicted by Sacherer. The beam envelope can be studied by calculating the second moment of the beam distribution over one turn of the ring. Figure 6 shows the one-turn second moments of the fully accumulated, high intensity beam, taken at three different longitudinal locations with respect to the rms longitudinal bunch length $\left(\sigma_{l}=\right.$ $1.66 \mathrm{rad}$, or $23.8 \mathrm{~m}$ ): one at approximately $0.01 \sigma_{l}$, one at approximately $0.25 \sigma_{l}$, and one at approximately $\sigma_{l}$. The moment is normalized by the zero-space-charge beam envelope, $\sqrt{\beta_{y}(s) \epsilon_{y}}$, such that the vertical axis represents a fractional deviation from this envelope. We observe that

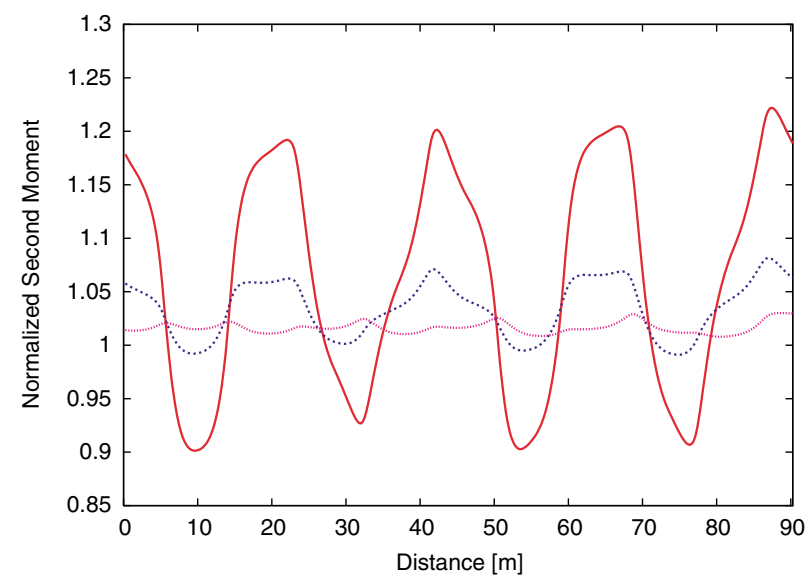

FIG. 6. (Color) Vertical second moment of the beam, normalized to $\sqrt{\beta_{y} \epsilon_{y}}$, taken over one turn near the end of accumulation of $4.37 \times 10^{13}$ protons. The curves correspond to longitudinal slices of beam at approximately $0.01 \sigma_{l}$ (red solid line), $0.25 \sigma_{l}$ (blue dashed line), and $\sigma_{l}$ (pink dotted line). the longitudinal center of the beam undergoes as much as a $20 \%$ deviation from the zero-space-charge envelope. The envelope executes about four oscillations per turn, consistent with Sacherer's predictions for the periodic, coherent half-integer envelope resonance. Notice also that the moment amplitudes drop very quickly outside of the longitudinal center. This behavior is detailed in Fig. 7, where the strength of the $m=4$ envelope harmonic is sampled via a Fourier transform of the second moments of the beam over the longitudinal coordinate range. As expected, the distribution of strengths is strongly peaked at the longitudinal center of the beam, with the harmonic strength dropping to less than half of its peak value within $0.2 \mathrm{rad}$, or $0.12 \sigma_{l}$, on either side of the longitudinal center. Therefore, the envelope resonance excitation of the PSR beam is heavily concentrated in the central, high density portion of the bunch. The effect of the sharply peaked longitudinal density distribution is discussed in more detail in Sec. IVA.

Figure 6 demonstrates that the resonant motion of the beam envelope is dominated by the periodic component, which is a fixed point in the envelope phase space. However, a real beam does not sit exactly on the fixed point, but instead executes small oscillations about this point [11,17]. The tune of the small oscillations is bounded above by the zero-space-charge upper limit, $\nu_{e}=2 \nu_{y}$, and decreases with increasing space charge. In the PIC simulations, the amplitude of the oscillation is too small to observe with second moment analysis. It is in fact much more practical to measure the tune of the mismatched envelope by solving the envelope equations of motion. For our calculations, we use an envelope model which has been derived from an rms standpoint and does not include higher order moment contributions [18]. The model has been implemented into the ACCSIM particle tracking code [19], where the envelope motion including

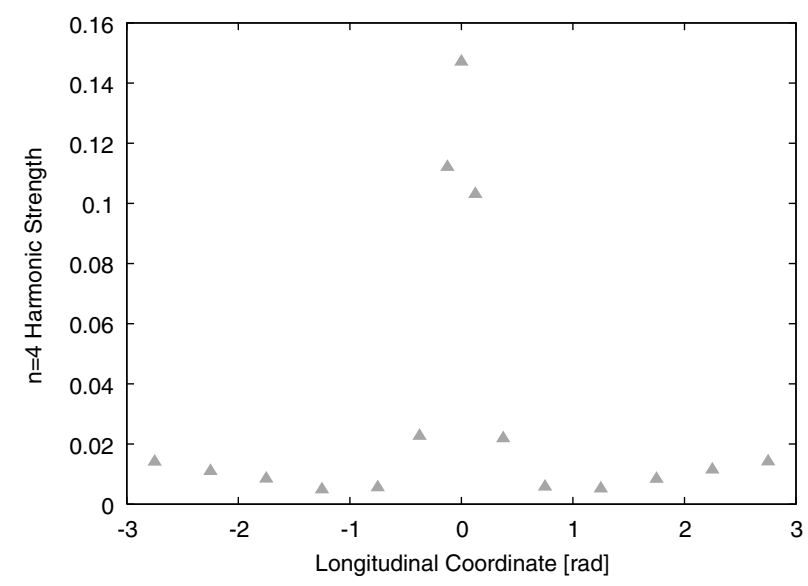

FIG. 7. Strength of the $\nu_{e}=4.0$ envelope harmonic versus longitudinal coordinate. The harmonic strengths are obtained via FFT of the second moments of the beam at specific longitudinal locations. 
dispersion is solved by iterating the following set of equations:

$$
\begin{gathered}
a^{\prime \prime}-\frac{\epsilon_{x}^{2}}{a^{3}}+\left[K_{x}(s)-\frac{4 q^{2} \lambda}{A(A+B) \gamma^{2} \beta^{2} E_{0}}\right] a=0, \\
b^{\prime \prime}-\frac{\epsilon_{y}^{2}}{b^{3}}+\left[K_{y}(s)-\frac{4 q^{2} \lambda}{B(A+B) \gamma^{2} \beta^{2} E_{0}}\right] b=0, \\
D_{x}^{\prime \prime}(s)+\left[K_{x}(s)-\frac{4 q^{2} \lambda}{A(A+B) \gamma^{2} \beta^{2} E_{0}}\right] D_{x}(s)-\frac{1}{\rho}=0 .
\end{gathered}
$$

Here, $a$ and $b$ are the envelope radii, $A=$ $\sqrt{a^{2}+4 D_{x}^{2} \delta^{2}}, B=b, D_{x}$ is the space-charge-adjusted dispersion function, $K_{x}(s)$ and $K_{y}(s)$ are the horizontal and vertical focusing functions, respectively, $\epsilon_{x}$ and $\epsilon_{y}$ are the horizontal and vertical emittances, $q$ is the proton charge, $\lambda$ is the beam particle line density, $\gamma$ and $\beta$ are relativistic factors, $E_{0}=\gamma m c^{2}$ is the reference energy of the beam, $\rho$ is the bending radius, $\delta=\Delta p / p$ is the rms momentum spread, and the derivatives are taken with respect to the longitudinal coordinate, $s$. The model assumes a coasting beam for the longitudinal distribution, which is a good approximation when the bunch length is long compared to the width of the beam, as is the case for the PSR machine.

Equations (1)-(3) describes the transport of the rms envelope of a fixed emittance, fixed intensity beam with momentum spread through a real accelerator lattice. The tune of the envelope at any point in the PSR accumulation can be derived from this model by using the intensity and rms emittance from the PIC calculation corresponding to the beam at that point of accumulation. Because the effect of the space charge is largest at the longitudinal center of the beam, for these calculations we use the emittance and intensity parameters for the central 5\% of the longitudinal beam distribution. The rms momentum spread of the beam was calculated from the PIC results and found to be $\Delta p / p \approx 0.002$. Additionally, to simulate the nearly matched condition of the PSR beam, a small initial envelope mismatch of $1 \%$ is assumed.

The results of the envelope tune calculations for the three different PSR beam intensity accumulation cycles are shown in Fig. 8. Recall that both the quarter and half intensity beams experience emittance growth due to injection painting effects alone. As the accumulated beam intensity increases, the envelope tunes for the quarter and half intensity beams both decrease, with the half intensity beam decreasing at a larger rate due to higher space charge forces. At the highest intensity, the envelope tune decreases for the first 1000 turns, and then remains nearly constant throughout the remainder of accumulation, despite the increasing beam intensity. Revisiting Fig. 4, we see that the beam emittance begins to grow in excess of

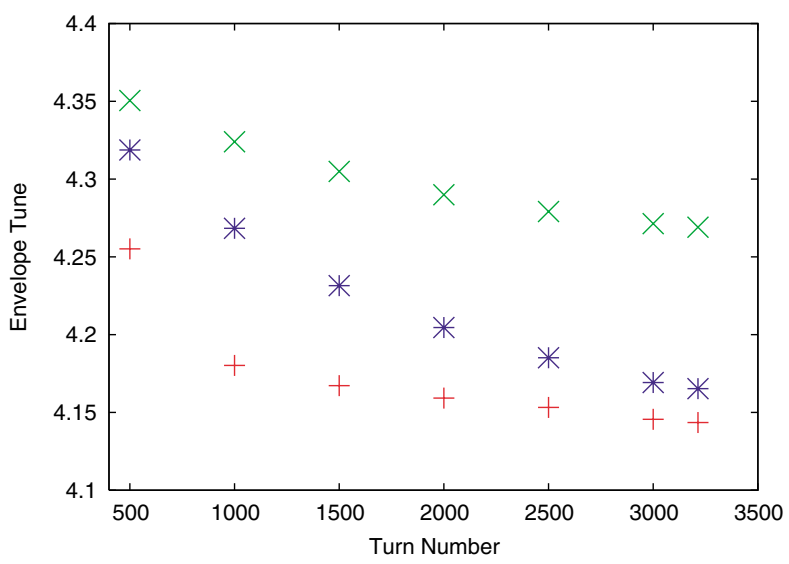

FIG. 8. (Color) Envelope tune (as calculated by an envelope model) versus turn number for the full intensity (red +'s), half intensity (blue *'s), and quarter intensity (green $X$ 's) PSR beam.

painting effects at about turn 1000. Therefore, the emittance of the beam grows to offset the effect of the intensity increase, and the envelope tune of the beam remains steady at $\nu_{e} \cong 4.14$. This result provides evidence of an envelope stop band surrounding $\nu_{e}=4.0$ for the PSR beam. In [11], the existence of a strong $n=$ 4 harmonic in the spectrum of the PSR vertical beta function was demonstrated. Aside from the PSR structure harmonics $(n=10,20,30, \ldots$,$) , this harmonic was$ shown to be the strongest harmonic in the vertical spectrum. The harmonic drives the periodic envelope resonant excitation (phase space fixed point) and the nonlinear structure of the entire phase space surrounding the fixed point. Thus, the driving term for the coherent half-integer resonance has been built into the lattice itself. The width of the observed stop band is related to the strength of the $n=4$ driving harmonic. Therefore, as will be demonstrated in Sec. V, a reduction of this harmonic should decrease the width of the stop band and reduce emittance growth in the PSR beam.

Continuing the investigation of the envelope dynamics, it is interesting to consider Sacherer's prediction that the resonant envelope amplitudes should increase nonlinearly with increasing beam intensity. Sacherer's analysis was predicated on the assumption of a constant beam emittance, and his results can been shown to hold for a beam with fixed emittance and increasing intensity $[5,11]$. If the emittance of the PSR beam is not constant, however, the evolution of the beam envelope cannot be accounted for by Sacherer's analysis. Figure 9 plots the maximum of the vertical second moment of the beam, normalized to the zero-space-charge envelope, $\sqrt{\beta_{y} \epsilon_{y}}$, over the course of the accumulation. We see that after 1500 turns, the amplitude of the resonant periodic envelope oscillation remains nearly fixed at about a $25 \%$ deviation from the zero-space-charge envelope. As with the envelope tune shift, the emittance growth of the beam prevents the 


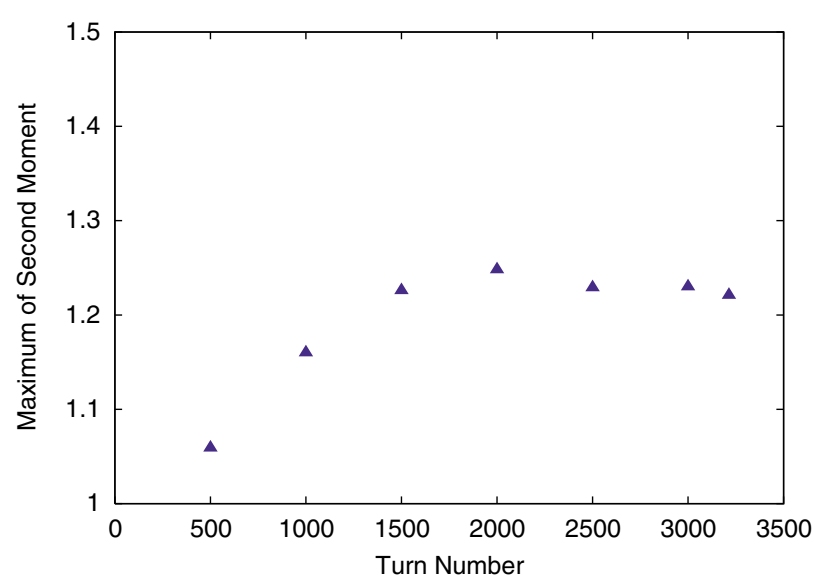

FIG. 9. (Color) PIC simulated maximum envelope oscillations (normalized to $\sqrt{\beta_{y} \epsilon_{y}}$ ) during accumulation of $4.37 \times 10^{13}$ protons in the PSR ring (colored points).

oscillation amplitude from increasing with intensity. Overall, we conclude that once the beam reaches the stop band, the emittance grows at the rate necessary to prevent the envelope dynamics from evolving with increasing beam intensity.

A brief comment should be made regarding the resonance condition in the horizontal plane. Slight beam broadening is sometimes observed in this direction as well, but the analysis of the phenomena is complicated by the fact that there is horizontal dispersion throughout the entire PSR machine. As shown in Ref. [11], the dispersion has two major effects on the resonance analysis: (1) it decreases the strength of the resonance response, and (2) it corrupts the resonance signal in the envelope oscillation, which cannot be decoupled from the dispersion function. For these reasons, it is difficult to clearly investigate the horizontal envelope resonance condition in PIC simulations. Envelope models can successfully treat idealized situations of this nature, but they cannot represent real experiments. We therefore do not dedicate any of the experiments in this paper to investigations of coherent resonances in the horizontal plane.

\section{BEAM SENSITIVITY TO VARIATIONS OF SPACE CHARGE ENVIRONMENT}

In addition to the beam intensity, the strength of the space charge in the beam depends on a number of parameters, including the longitudinal bunching factor, the bare lattice tune, and the beam emittance. We present here three experiments that investigate the sensitivity of the beam to variations of these quantities. In each experiment, exactly one of the aforementioned parameters is varied, and as before the resonant response of the beam is assessed through experimental profile measurements, PIC simulations, and envelope calculations. As always, the PIC simulations were certified by benchmark with mea- sured data in advance of incorporating the results into the analysis.

\section{A. Variation of longitudinal bunch shape}

An important result of the previous section was that the sharply peaked center of the longitudinal distribution contains the bulk of the strongly resonant portion of beam. The shape of the distribution is determined by the rf cavity and the injection scenario, but some additional influence can be exerted by activating a $12 \mu \mathrm{H}$ inductor coil inside the PSR ring. The inductor coil was placed in the ring to provide longitudinal space charge compensation and to reduce the beam in the gap [20]. Though the coil has proved effective at this task, the bunch compression realized from the inductance increases the density of the longitudinal distribution, especially near the center of the bunch train (see Fig. 2). As a means of relieving the problem, an experiment was conducted to alter the longitudinal distribution by inserting a zero-beam notch at the very center of the bunch train. Specifically, no beam was injected within $30 \mathrm{~ns}\left( \pm 15^{\circ}\right)$ of the center of the bunch train; additional beam was injected outside of this range so that the overall intensity of the beam was unaffected. All other parameters in the experiment remained as in Table I.

Figure 10 shows the experimentally measured longitudinal beam profiles after the full accumulation of $4.37 \times$ $10^{13}$ protons for beams with and without the longitudinal notch. Although the beam eventually migrates into the notched region, the overall peaking is greatly reduced, and the bunching factor, defined as $I_{\text {average }} / I_{\text {peak }}$, increases from the nominal value of approximately 0.30 to approximately 0.45 . The effect of the notch on the transverse plane is shown in Fig. 11, where the experimental vertical beam profiles are shown for the corresponding notched and non-notched beams. The notch reduces the amount

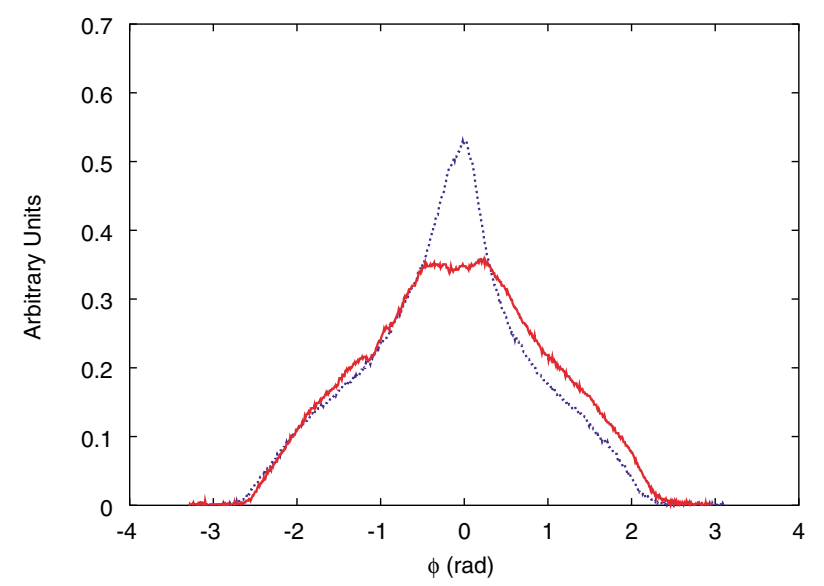

FIG. 10. (Color) Experimental longitudinal profile measurements. The blue curve is the profile of the beam without the longitudinal notch, and the red curve is the profile of the beam with the longitudinal notch. 


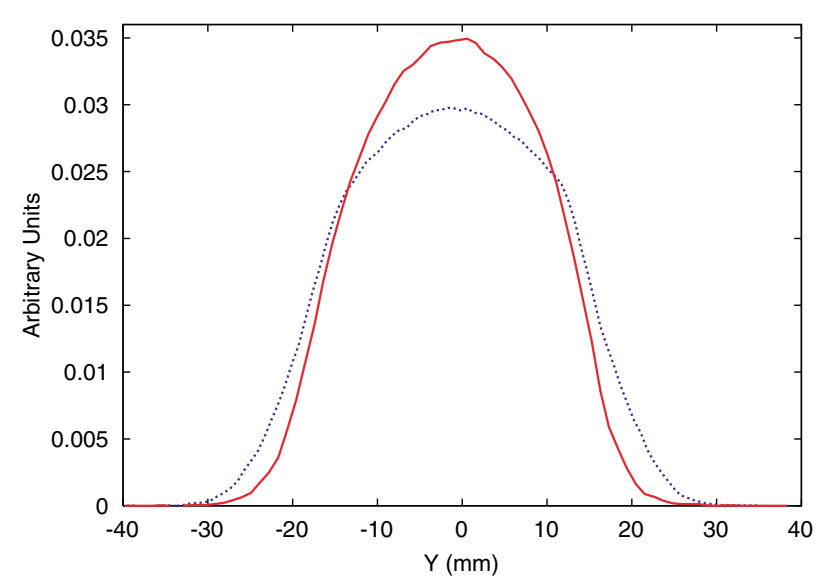

FIG. 11. (Color) Experimental transverse vertical profile measurements of the full intensity beam. The (blue) dashed curve is the profile of the beam without the longitudinal notch, and the (red) solid curve is the profile of the beam with the longitudinal notch.

beam broadening induced, with the notched beam approximately $15 \%$ thinner than the non-notched beam. To confirm that the reduction in broadening is linked with a weakening of space charge forces and not another phenomenon, Fig. 12 shows experimentally measured profiles for notched and non-notched half intensity beams. It has been previously demonstrated that the beam undergoes little space-charge-induced emittance growth at this intensity, and as such, we observe that the notch has no effect on the transverse profile in this case.

The tune space of the particles is altered significantly by the beam notch. Figure 13 shows the incoherent tune footprint versus longitudinal coordinate for the notched and non-notched high intensity beams. It is apparent that

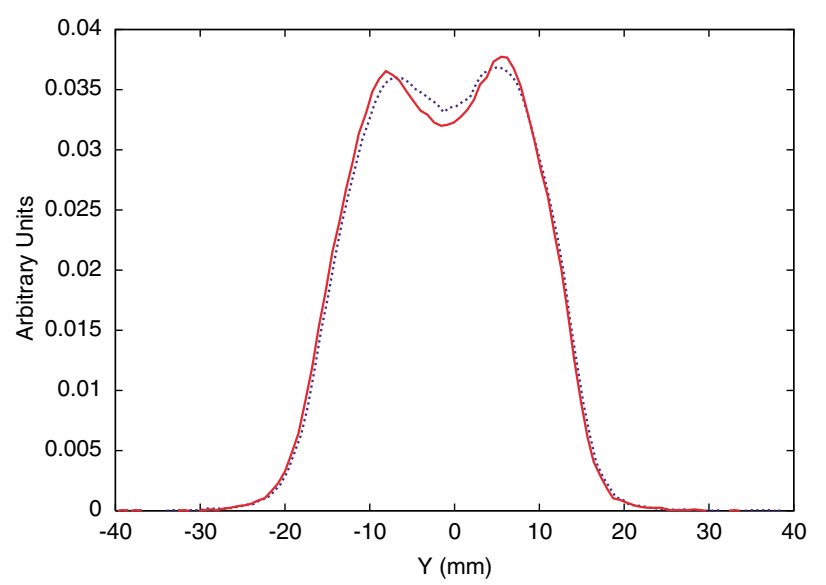

FIG. 12. (Color) Experimental transverse vertical profile measurements of the half intensity beam. The (blue) dashed curve is the profile of the beam without the longitudinal notch, and the (red) solid curve is the profile of the beam with the longitudinal notch.

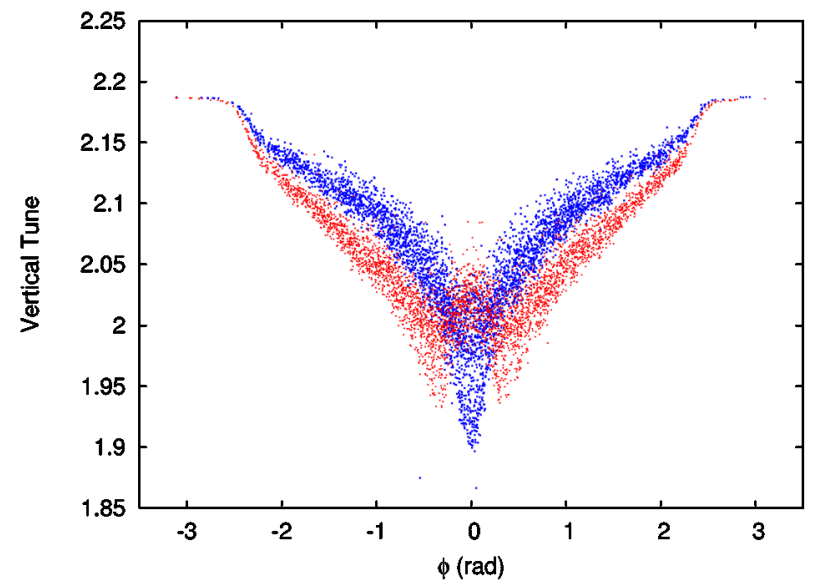

FIG. 13. (Color) Incoherent particle tunes taken from PIC simulation versus longitudinal coordinate for the high intensity beam. The blue points represent the beam without the longitudinal notch, and the red points represent the beam with the longitudinal notch.

the notch reduces the space charge tune depression in the center of the bunch train, bringing the maximally depressed tunes from $\nu_{y} \approx 1.9$ back up to $\nu_{y} \gtrsim 2.0$. The strongest space charge region now lies just outside of the notch, where the maximally depressed particle tunes are still somewhat below the integer value.

The benefit of the notch manifests itself clearly through the emittance parameter. Figure 14 tracks the rms vertical beam emittance for the notched and nonnotched full and half intensity beams over the accumulation cycle. As expected, emittance growth at the high intensity is reduced considerably by the beam notch, with the final emittance of the notched beam over $25 \%$ smaller than the emittance of the non-notched beam; no

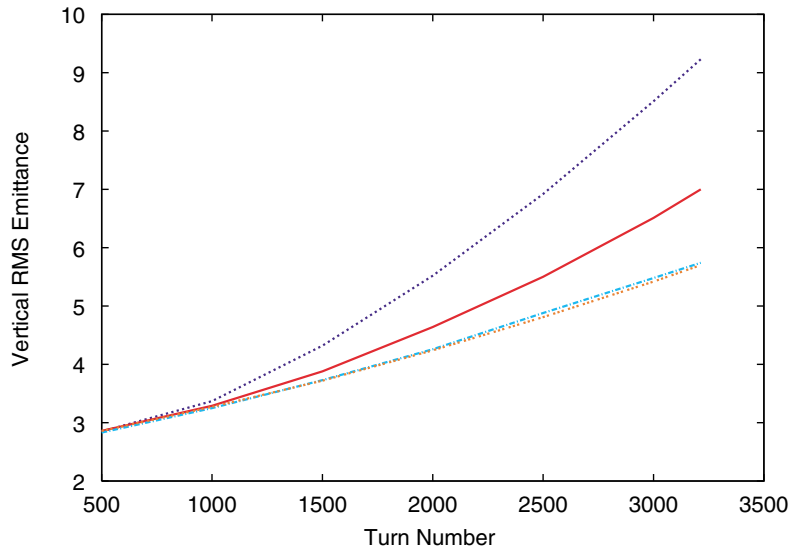

FIG. 14. (Color) Simulated vertical rms beam emittances for the notched and non-notched beams during accumulation of full and half intensity beams. The red solid and orange dashed lines are the notched beams at full and half intensity, respectively, and the blue dotted and turquoise dashed lines are the non-notched beams at full and half intensity, respectively. 
difference in emittance behavior is observed in the half intensity beams. The fact that the emittance of the beam grows in both high intensity cases indicates that the envelope tune still reaches the stop band, even in the notched beam case. However, the space charge force is less in the notched beam, and the emittance does not have to grow as much to balance the increase of intensity.

\section{B. Variation of vertical bare tune}

Probably the most obvious control variable for the beam resonant response is the bare tune of the lattice. The PSR lattice is flexible to changes in the bare tune in the range of $\left(\nu_{x}, \nu_{y}\right)=(1.5-4.0,1.5-4.0)$. However, higher order structure resonances and losses near the extraction region constrain the working point to remain near the baseline value of $\left(\nu_{x}, \nu_{y}\right)=(3.23,2.23)$. For the following experiment, the vertical bare tune was varied from the nominal value of 2.19 down to 2.09, while keeping the horizontal tune fixed. Experimental measurements were taken for $\nu_{y}=2.19,2.15,2.13,2.11$, and 2.09. The other experimental parameters are listed in Table II. Note that the beam intensity used here is lower than that of the experiments presented in Sec. III.

Figure 15 shows the experimental vertical beam profiles after the full accumulation of beam over the range of bare tune values. The result of the tune variation is an increase of the full beam width from approximately $17 \mathrm{~mm}$ at the highest bare tune to $22 \mathrm{~mm}$ at the lowest bare tune. Figure 16 shows the experimentally measured beam loss in the ring for the different bare tune values. The data are digitized sums of beam loss readings from ion chambers located around the ring [21], with an absolute measurement error of $\leq 15 \%$, and a smaller relative error. At the lowest bare tune value $\left(\nu_{y}=2.09\right)$, the experimental beam losses jump and it is clear that the beam emittance has exceeded the machine aperture. Because of radiation safety concerns, it was not possible during the experiment to lower the vertical tune below $\nu_{y}=2.09$.

It is insightful to examine the envelope tunes throughout the accumulation of the beams with the different bare tune values. Figure 17 shows the result, where again the envelope tunes have been calculated using the PICsimulated emittances and intensities in the envelope model and assuming a small initial mismatch (1\%). For

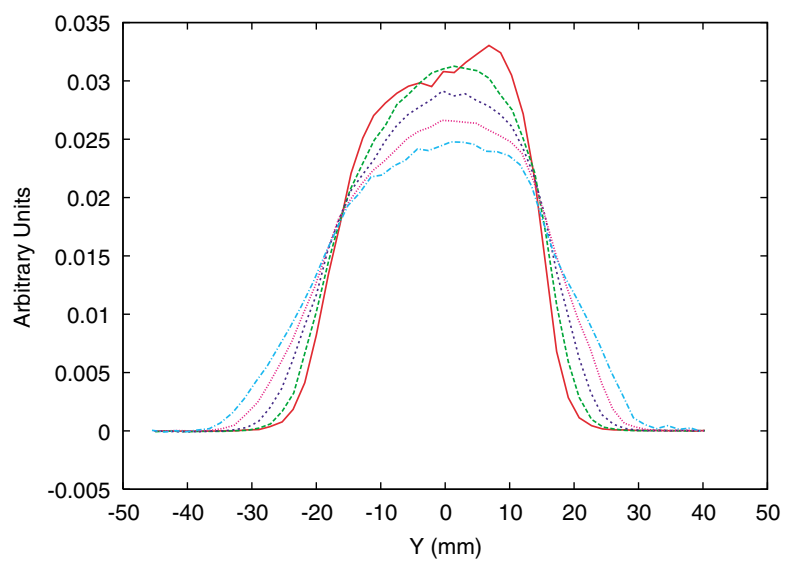

FIG. 15. (Color) Experimentally measured vertical beam profiles for tunes in the range of $\left(\nu_{x}=3.19, \nu_{y}=2.09, \ldots, 2.19\right)$, where the profile widths increase as $\nu_{y}$ decreases from 2.19 to 2.09 (2.19, 2.15, 2.13, 2.11, 2.09).

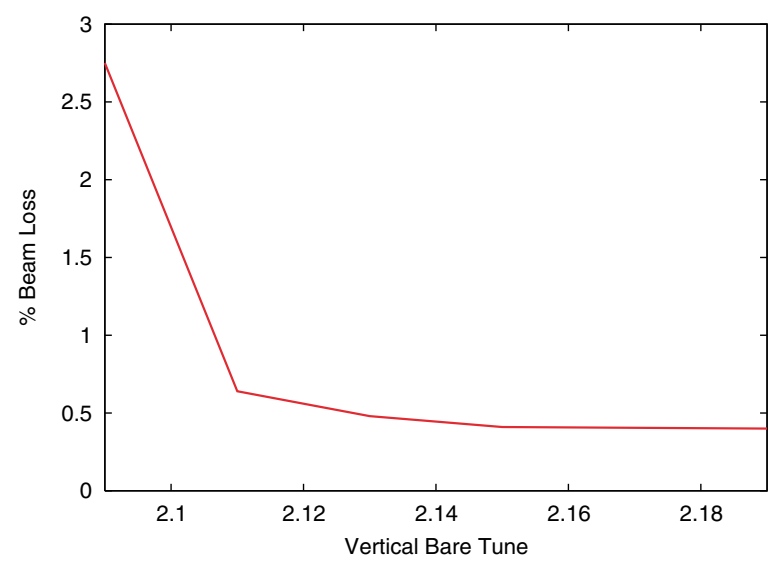

FIG. 16. (Color) Experimentally measured beam loss during accumulation versus vertical bare tune.

the highest value of the bare tune $\left(\nu_{y}=2.19\right)$, the envelope tune begins to settle out only at the end of the accumulation cycle. In this case, the beam adiabatically approaches the stop band edge, which is a function of the lattice parameters. For the two lower bare tunes, the envelope is much closer to the stop band at the beginning of accumulation and it encounters the condition very quickly, especially for the case with $\nu_{y}=2.09$. Note

TABLE II. Parameters for the Tunescan Experiment.

\begin{tabular}{lc}
\hline \hline Full beam intensity & $3.13 \times 10^{13}$ protons \\
Injection period & $1.225 \mathrm{~ms}$ \\
Linac beam horizontal offset & $x=4.77 \mathrm{~mm} ; x^{\prime}=-0.61 \mathrm{mrad}$ \\
Linac beam vertical offset & $y=16.47 \mathrm{~mm} ; y^{\prime}=2.65 \mathrm{mrad}$ \\
Vertical closed orbit bump at foil & $12.0 \rightarrow 0 \mathrm{~mm}$, and $1.65 \rightarrow 0 \mathrm{mrad}$ \\
Bare lattice tunes & $\left(\nu_{x}=3.19, \nu_{y}=2.09, \ldots, 2.19\right)$ \\
rf voltage & $11.113 \mathrm{kV}$ \\
\hline \hline
\end{tabular}




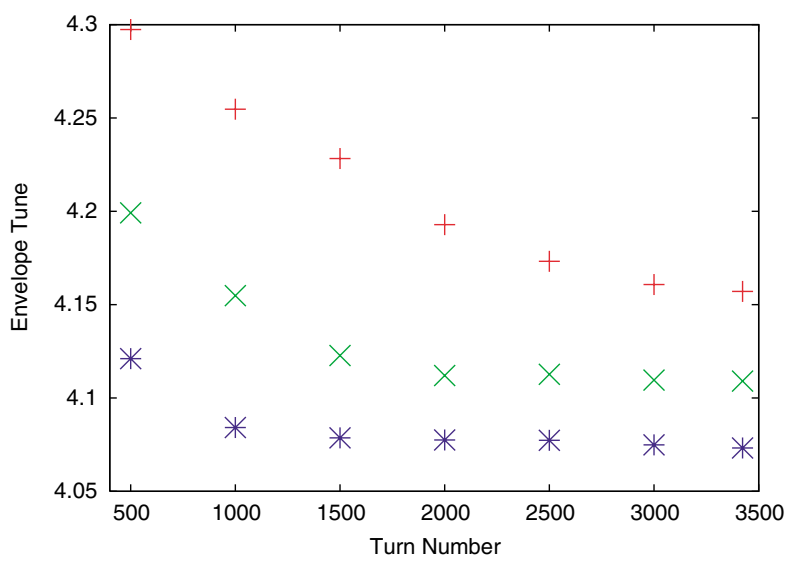

FIG. 17. (Color) Envelope tune (as calculated by a PCM model) versus turn number for $\nu_{y}=2.19$ (red +'s), $\nu_{y}=2.13$ (green $\times$ 's), and $\nu_{y}=2.09$ (blue $*$ 's).

that there is some difference in the saturated value of the envelope tune for the three bare tune cases. This implies that the stop band has a soft edge which can be penetrated by forcefully driving the beam into the stop band. For any given working point and injection scheme, the driven steady-state envelope tune is attained when the rate of emittance growth balances the rate of accumulation to provide a constant space charge environment. Thus, the driven steady-state envelope tune is a function of the machine working point and the space charge force in the beam. To further illustrate this, we consider the evolution of the second moment oscillations, shown in Fig. 18. The figure indicates that the steady-state oscillation amplitude obtained by the beam is much larger for lower bare tune values. Additionally, as shown in Fig. 19, maintaining the lower envelope tune and larger envelope oscillations requires a significantly higher rate of emittance growth than does maintaining the higher envelope tune and smaller oscillations.

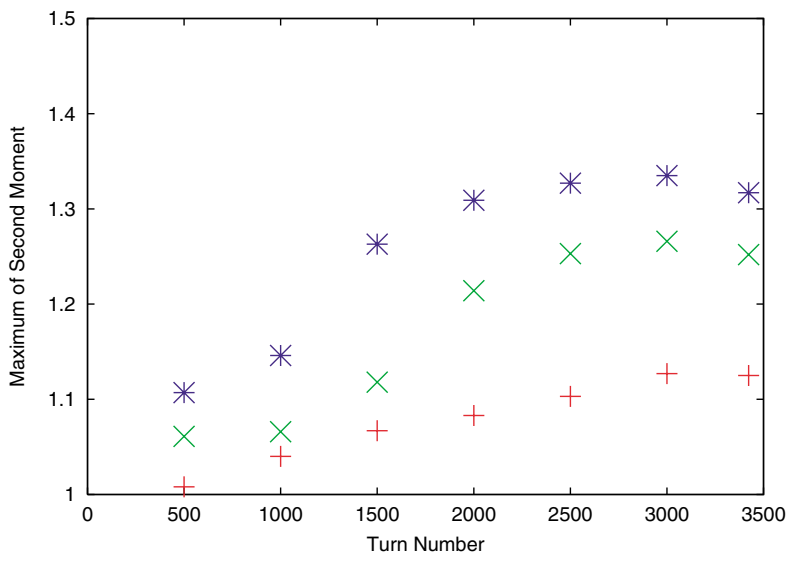

FIG. 18. (Color) PIC simulated maximum envelope oscillations (normalized to $\sqrt{\beta_{y} \epsilon_{y}}$ ) versus turn number for $\nu_{y}=$ 2.19 (red +'s), $\nu_{y}=2.13$ (green $\times$ 's), and $\nu_{y}=2.09$ (blue $*$ 's).

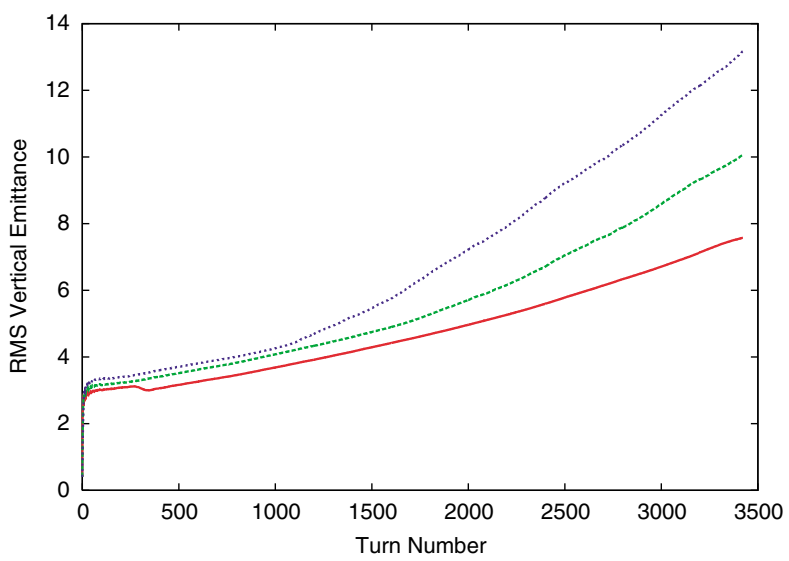

FIG. 19. (Color) Vertical emittance evolution for $\nu_{y}=2.19$ (red solid line), $\nu_{y}=2.13$ (green dashed line), and $\nu_{y}=2.09$ (blue dotted line).

To test the hypothesis that the saturated envelope state depends on how hard the beam is forced into the stop band, a simulation was performed to double the rate of injection for the beam with bare tune $\nu_{y}=2.13$. In this case, the full PSR beam intensity is accumulated in half the number of turns (about 1500). The resulting envelope tunes are shown in Fig. 20, along with those observed for the normal injection rate. As expected, the beam driven envelope steady state is a function of the space charge force in the beam, and the case with double the injection rate reaches much farther into the stop band than the case with the normal injection rate.

As a means of locating the edge of the stop band, we simulated the effect of storing the beams with bare tunes $\nu_{y}=2.19,2.13$, and 2.09 for 1000 turns beyond accumulation. The result is shown in Fig. 21. Recall that for the highest bare tune value, the beam reaches a steady-state envelope tune at the very end of accumulation (see Fig. 17).

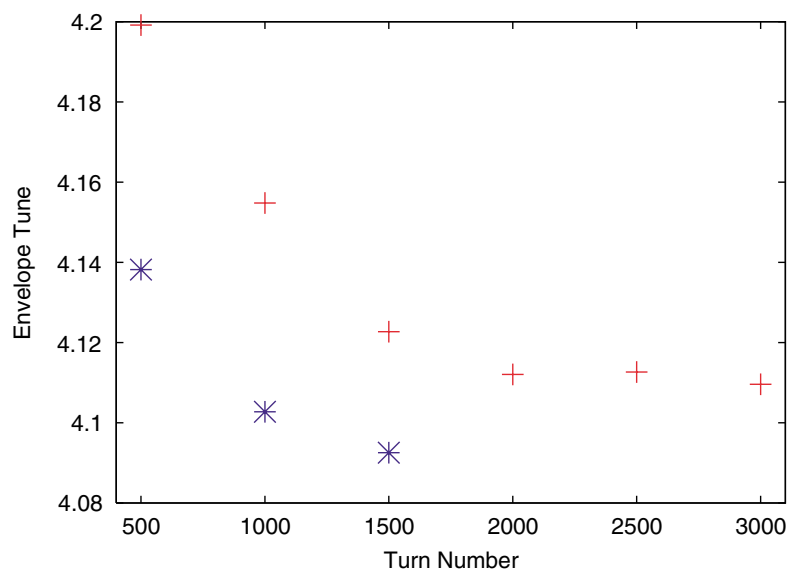

FIG. 20. (Color) Envelope tune (as calculated by a PCM model) versus turn for the lattice setting $\left(\nu_{x}, \nu_{y}\right)=$ $(3.19,2.13)$ for the regular PSR beam injection rate (red +'s), and for twice the regular injection rate (blue $*$ 's). 


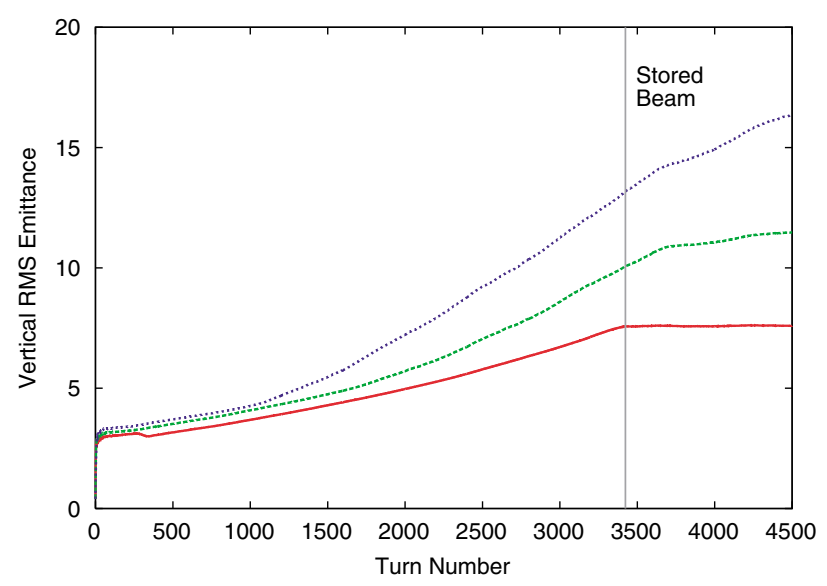

FIG. 21. (Color) Simulated vertical rms emittances versus turn for the full accumulation of the beam, followed by 1000 turns of beam storage. The solid (red) line shows the case for $\nu_{y}=$ 2.19 , the dashed (green line shows the case for $\nu_{y}=2.13$, and the dotted (blue) line shows the case for $\nu_{y}=2.09$. The dashed vertical line marks the end of accumulation at 3423 turns.

Since no emittance growth is seen during the storage, we can conclude that the edge of the stop band lies near $\nu_{e} \approx$ 4.14. A beam which approaches the stop band slowly will remain with the envelope tune close to this value during storage. For the two lower bare tune values, where the driven steady-state envelope tune is below this limit, the emittance growth of the stored beam continues past the time that the intensity accumulation has ceased; the envelope tune is slowly rising back up to the edge of the stop band. However, for the emittance growth rates exhibited in the plot, several thousand turns would be necessary before the beam would reach the edge of the stop band and the emittance growth would cease.

\section{Variation of painted beam emittance}

In the following experiment, the strength of the space charge force in the beam is varied by changing the injection painting scheme. Two beams with different linac injection offsets were painted: one with a large, $17.51 \mathrm{~mm}$ injection offset, and the other with a small, $12.61 \mathrm{~mm}$ injection offset. The remainder of the experimental parameters is given in Table III. Each of the beams

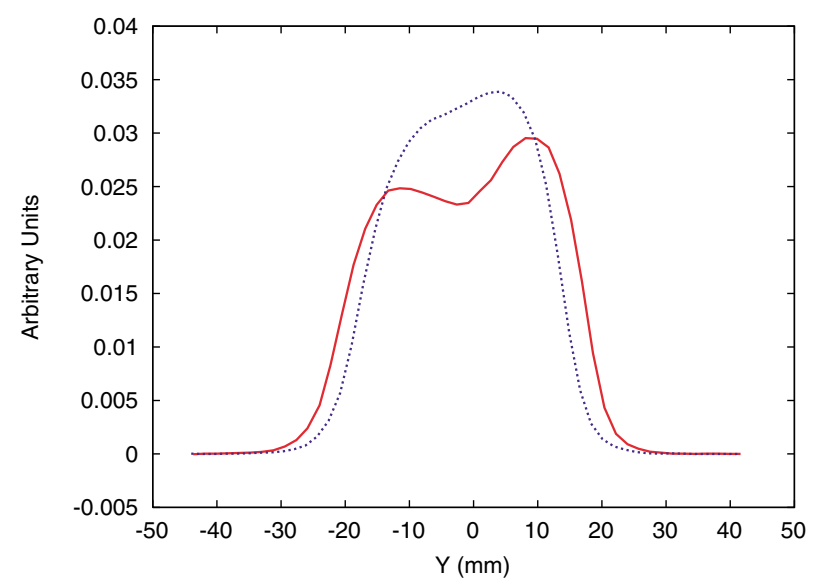

FIG. 22. (Color) Experimentally measured vertical beam profiles for two different painting schemes of a low intensity beam. The red curve is the beam with a $17.51 \mathrm{~mm}$ injection offset, and the blue curve is the beam with a $12.61 \mathrm{~mm}$ injection offset.

was accumulated with full and half beam intensities. The half intensity cases are shown in Fig. 22, where the measured vertical profiles for both painting schemes are presented. In accord with the painting scheme, the smaller injection-offset beam remains smaller throughout the accumulation cycle, and space charge has no obvious influence on the profile distribution. At the full intensity, shown in Fig. 23, we find that both beams undergo beam broadening and have approximately the same profile distribution at the end of accumulation. The simulated emittances of the high intensity beams are shown in Fig. 24, along with the calculated envelope tunes. As seen, the smaller, more space charge intense beam reaches the envelope stop band more quickly and undergoes a larger amount of emittance growth overall.

Under the influence of the space charge effect, the final distribution of the high intensity beam is nearly independent of the injection scheme. It is thus very difficult to exercise control over the beam distribution when space charge forces are strong. It should however be possible to control the rate of emittance growth in the beam by reducing the strength of the lattice harmonic which drives the integer stop band. A simple example of the effect of the stop band correction is discussed in the next section.

TABLE III. Parameters for the injection painting experiment.

\begin{tabular}{lc}
\hline \hline Full beam intensity & $3.13 \times 10^{13}$ protons \\
Injection period & $1.225 \mathrm{~ms}$ \\
Linac beam horizontal offset & $x=4.77 \mathrm{~mm} ; x^{\prime}=-0.61 \mathrm{mrad}$ \\
Linac beam vertical offset, large beam & $y=17.51 \mathrm{~mm} ; y^{\prime}=3.0 \mathrm{mrad}$ \\
Linac beam vertical offset, small beam & $y=12.61 \mathrm{~mm}, y^{\prime}=2.1 \mathrm{mrad}$ \\
Vertical closed orbit bump at foil, large beam & $12.0 \rightarrow 0 \mathrm{~mm}$, and $1.65 \rightarrow 0 \mathrm{mrad}$ \\
Vertical closed orbit bump at foil, small beam & $8.0 \rightarrow 0 \mathrm{~mm}$, and $1.1 \rightarrow 0 \mathrm{mrad}$ \\
Bare lattice tunes & $\left(\nu_{x}, \nu_{y}\right)=(3.19,2.19)$ \\
rf voltage & $11.113 \mathrm{kV}$ \\
\hline \hline
\end{tabular}




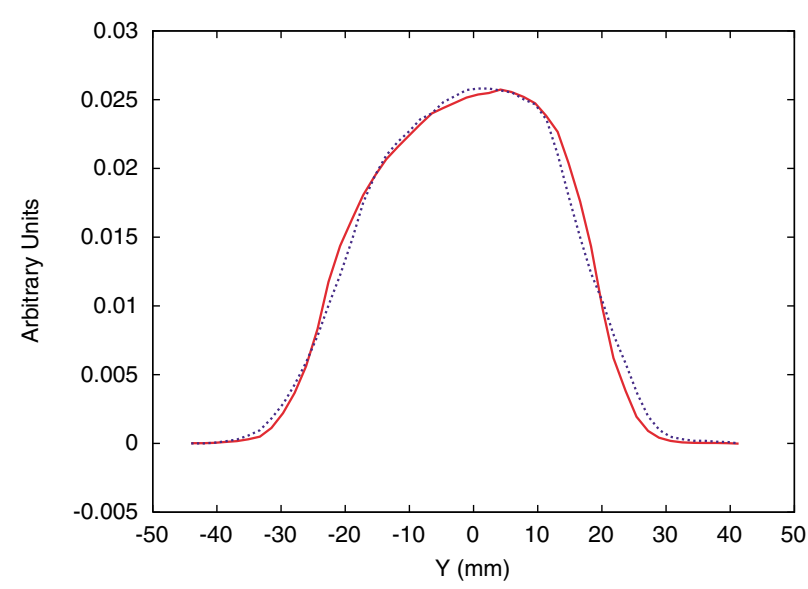

FIG. 23. (Color) Experimentally measured vertical beam profiles for two different painting schemes of a high intensity beam at the end of accumulation. The (red) solid curve is the beam with a $17.51 \mathrm{~mm}$ injection offset, and the (blue) dashed curve is the beam with a $12.61 \mathrm{~mm}$ injection offset.

\section{STOP BAND CORRECTION}

The PSR lattice is a ten-fold lattice with broken symmetry. The broken symmetry creates a number of low-order harmonics that can act as driving terms for envelope resonant excitations. For typical PSR bare tunes, the vertical envelope stop band is driven by the $n=4$ lattice harmonic. An effective correction should alleviate some or all of the emittance growth associated with the harmonic.

In the most extreme scenario, all of the lattice harmonics can be removed from the ring by using a phaseadvance-equivalent uniform focusing lattice. For this lattice, the focusing function is a constant throughout the ring, adjusted to provide particle phase advances in each plane that are identical with the real PSR lattice. Thus, for the uniform focusing case, there are no lattice harmonics. In the simulation, the injection offset and closed orbit bump were adjusted to produce the same vertical and horizontal beam emittance evolutions as the real PSR lattice in the zero-space-charge limit. All other parameters remained as in Table I. The results of the vertical emittance evolution for the uniform lattice case are shown in Fig. 25. Comparing with the lowest intensity case in Fig. 4, we see that the slow emittance growth observed in the vertical direction is due to painting effects alone. In the absence of any driving harmonics, there is no space-charge-induced emittance growth of the beam.

In a somewhat more realistic demonstration of the idea, the fundamental superperiod of the PSR lattice was identified and used to construct a perfectly symmetric tenfold PSR-like lattice. The vertical harmonic spectrum of the symmetric lattice is shown in Fig. 26, along with the harmonic spectrum of the real PSR lattice, both for working tunes $\left(\nu_{x}, \nu_{y}\right)=(3.19,2.19)$. In the symmetric
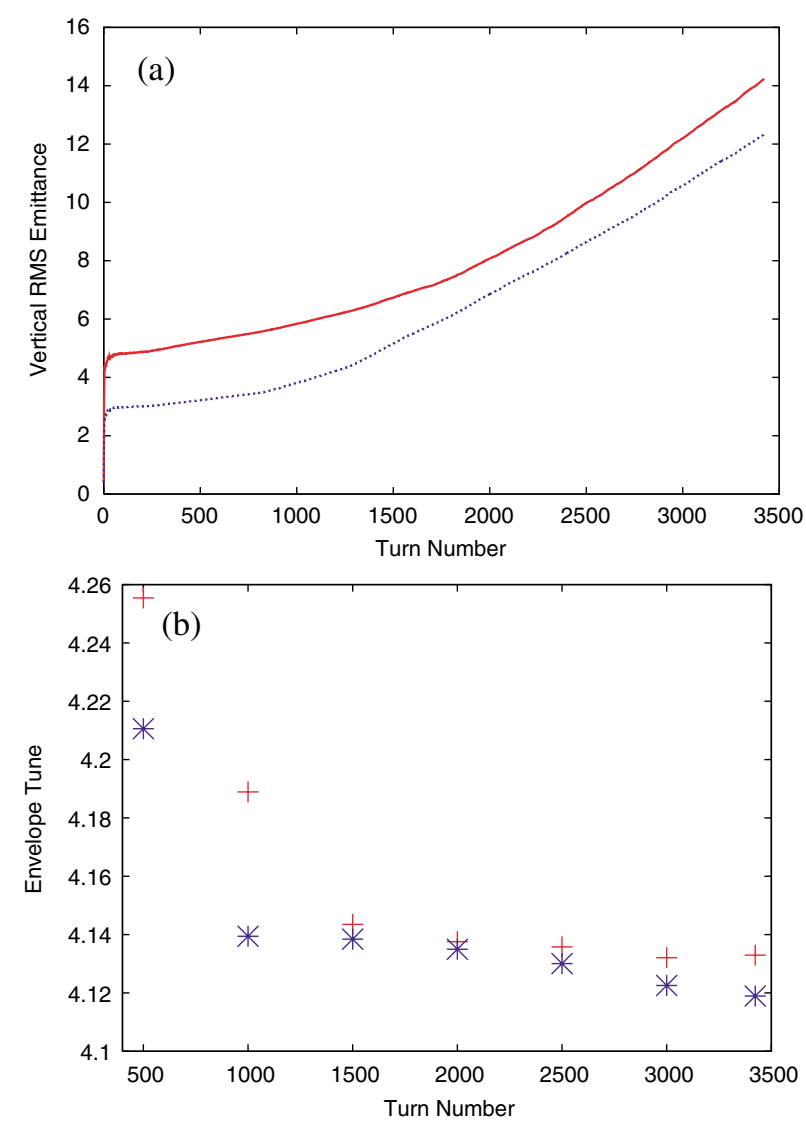

FIG. 24. (Color) Top panel: Simulated vertical rms emittances during the accumulation of a high intensity beam with two different injection painting schemes. The solid (red) curve represents the beam with a $17.51 \mathrm{~mm}$ injection offset, and the dashed (blue) curve represents the beam with a $12.61 \mathrm{~mm}$ injection offset. Bottom panel: Calculated envelope tune versus turn for the $17.51 \mathrm{~mm}$ injection offset (red +'s), and for the $12.61 \mathrm{~mm}$ injection offset (blue *'s).

lattice, all of the lower order harmonics caused by the broken symmetry have been eliminated, and only the structure harmonics remain. Note that in the real PSR lattice, the $n=4$ harmonic is the strongest nonstructure harmonic in the vertical plane.

A simulation of a high intensity PSR accumulation scenario was performed using the symmetric lattice and parameters as in Table I. The evolutions of the vertical rms beam emittance both with and without space charge are shown in Fig. 27. For comparison, the results of the simulation using the real PSR lattice are also shown. For the real PSR lattice, the final vertical rms emittance of the beam is about $60 \%$ greater than the zero-space-charge case. On the other hand, for the case with the symmetric lattice, the rms vertical emittance growth is only about $10 \%$ greater than the zero-space-charge case. However, it should be noted that prior to the upgrade of the PSR for $\mathrm{H}^{-}$injection, the lattice was more symmetric, and emittance growth was still observed for small beams or when the bare vertical tune was below $\nu_{y}=2.10$ [22]. The 


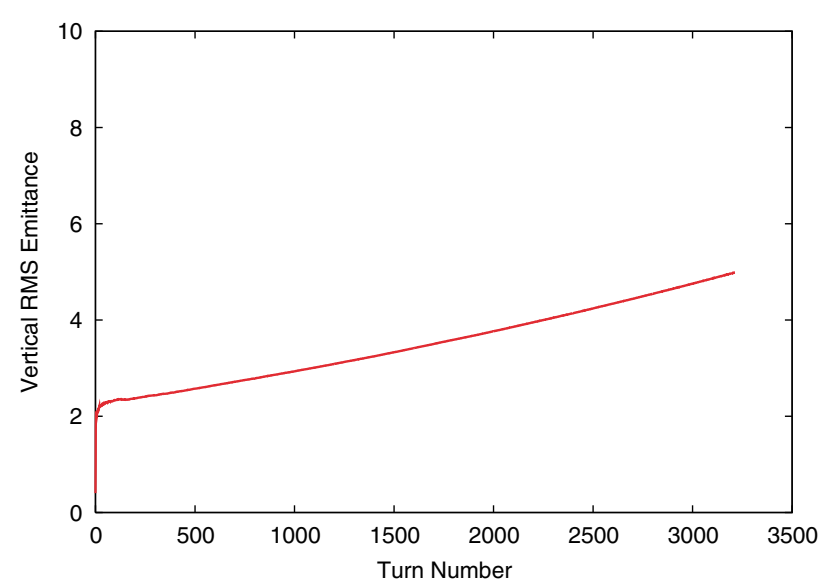

FIG. 25. (Color) Simulated vertical rms emittances during the accumulation of a high intensity beam in a PSR phaseadvance-equivalent, uniform focusing lattice.

removal of the driving term for the stop band can result in a significant decrease in the emittance growth the beam experiences, but some emittance growth should still be expected from the presence of the structure harmonics.
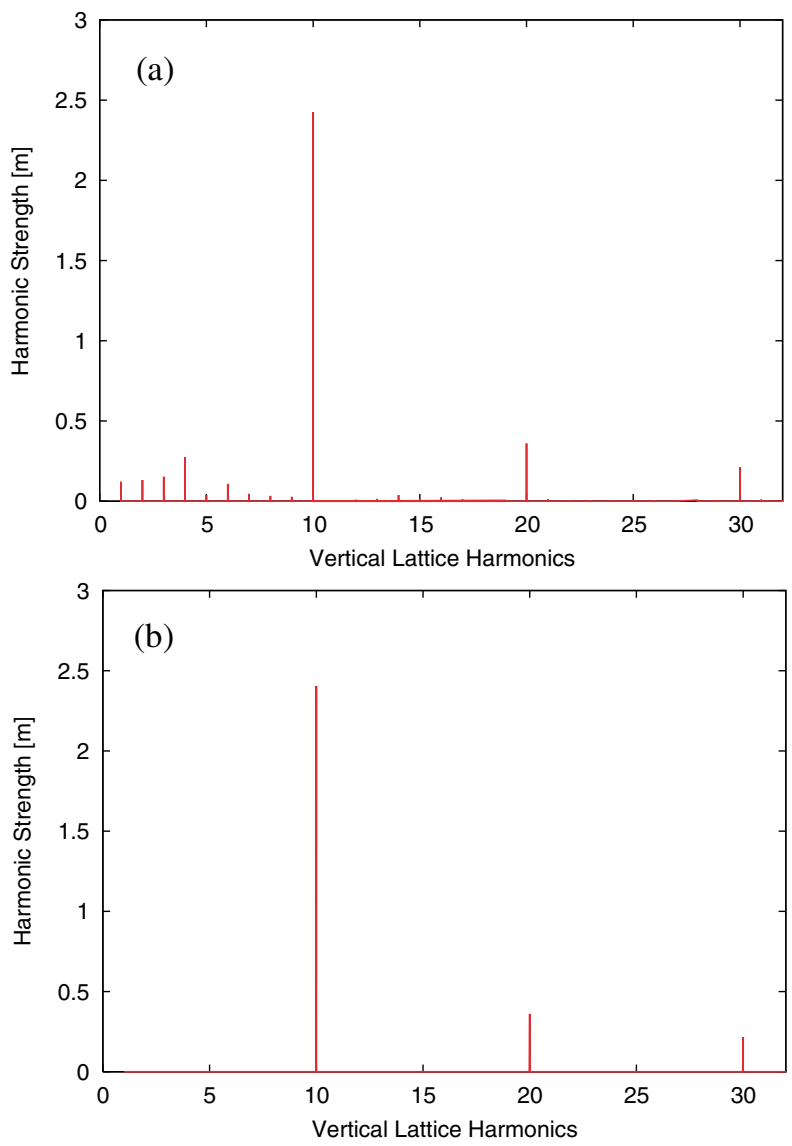

FIG. 26. (Color) Top panel: Harmonic spectrum of the vertical beta function for the real PSR lattice. Bottom panel: Harmonic spectrum of the vertical beta function for a perfectly symmetric, PSR-like lattice. Both lattices have $\left(\nu_{x}, \nu_{y}\right)=(3.19,2.19)$.
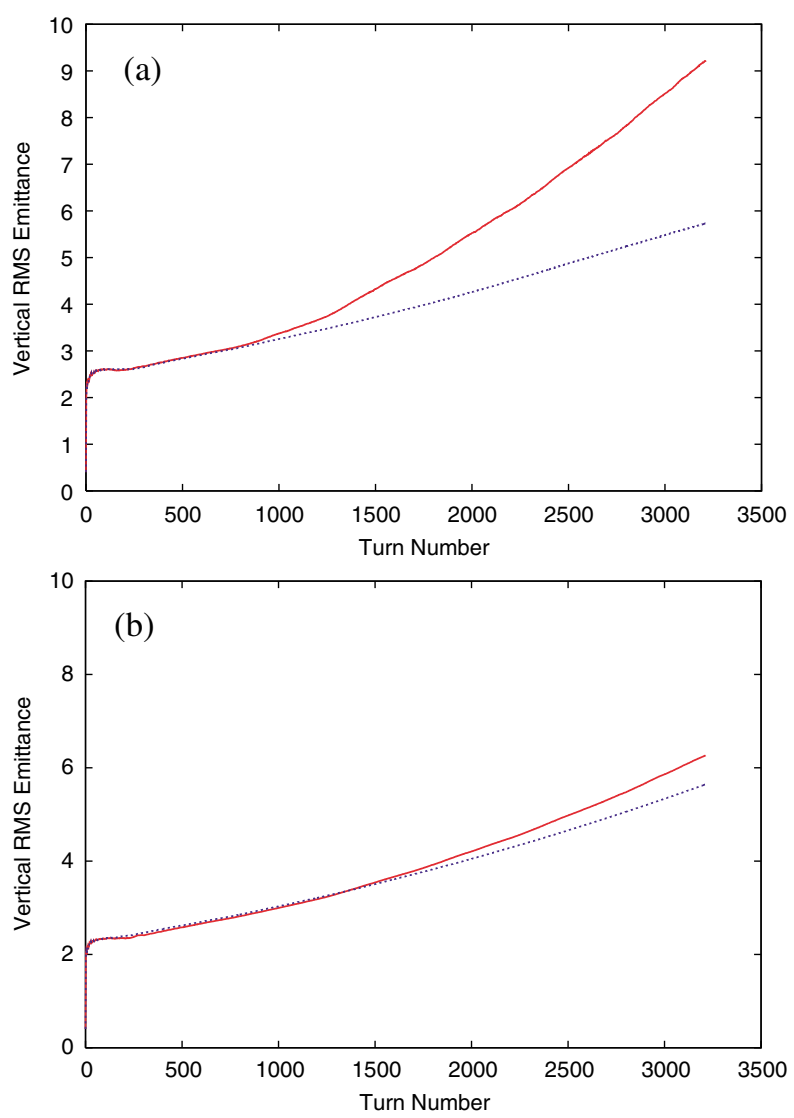

FIG. 27. (Color) Top panel: Vertical rms emittance versus turn number during the accumulation of a high intensity beam in the real PSR lattice. Bottom panel: Vertical rms emittance versus turn number during the accumulation of a high intensity beam in the symmetric PSR-like lattice. In both plots, the solid (red) curves represent the beam with space charge, and the dashed (blue) curves represent the beam without space charge.

Recall that in the case of the uniform focusing lattice, where no lattice harmonics are present, there was no space-charge-induced emittance growth.

An examination of the incoherent tune footprint of the beam shows the extent to which the stop band correction affects the strength of the space charge in the beam. The footprints for symmetric lattice and the real PSR lattice are compared in Fig. 28. The particle tunes are maximally depressed to $\nu_{y}=1.9$ in the real lattice and to $\nu_{y}=$ 1.8 in the symmetric lattice. The emittance growth in the real lattice case weakens the space charge forces, thus preventing the particle tunes from depressing beyond $\nu_{y}=1.9$, whereas in the case of the symmetric lattice, the smaller emittance means stronger space charge forces and lower tunes.

The result of the symmetric lattice calculation is a bestcase scenario for the stop band correction. In reality it is not possible to create a perfectly symmetric storage ring, nor is it possible to correct all of the lower harmonics at once. However, the example demonstrates the potential benefit of a stop band correction. A real correction 


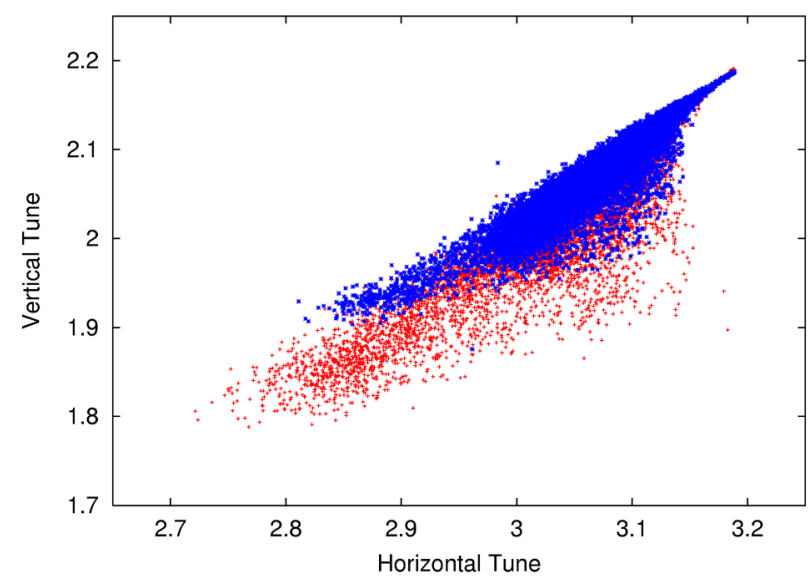

FIG. 28. (Color) Tune footprints of the beam in the real PSR lattice (blue points), and the symmetric, PSR-like lattice (red points). The bare tune for both cases is $\left(\nu_{x}, \nu_{y}\right)=(3.19,2.19)$.

scheme would aim to reduce or eliminate only the $n=4$ harmonic in the lattice, without significantly increasing neighboring harmonics. The correction could significantly impact the operating intensity of the PSR machine.

\section{CONCLUSIONS AND DISCUSSION}

We have studied the systematics of the vertical beam broadening in the PSR ring at high intensities. We find through simulations of several experiments that the space-charge-depressed particle tunes cross the incoherent stop band before the onset of any beam broadening, and that the coherent motion of the beam oscillates about the zero-space-charge betatron envelope with nearly four oscillations per turn; the strongest oscillations occur within a few degrees about the longitudinal center of the beam and drop sharply outside of this range. Furthermore, we find through a combination of PIC simulations and envelope model calculations that the beam encounters an envelope stop band when the coherent tune approaches $\nu_{e} \cong 4.14$ (for the PSR lattice settings used in this work). The onset of emittance growth occurs when the beam reaches the edge of the stop band and continues as long as the beam intensity increases. The rate of emittance growth balances the intensity increase to maintain driven steady-state envelope dynamics (tunes and resonant oscillation amplitudes).

Through three experiments that varied key beam parameters, we explored the systematics of the beam response to the stop band. It was shown that the stop band is soft edged. Furthermore, a beam which is forcefully driven into the stop band will undergo emittance growth after the intensity accumulation has ceased, whereas a beam which slowly reaches the edge of the stop band during accumulation will not undergo further emittance growth without an increase of beam intensity.

The potential effect of a stop band correction in the PSR lattice has also been studied. Using an artificially constructed, perfectly symmetric PSR-like lattice, it has been shown that the removal of the harmonic term that drives the envelope stop band results in a near elimination of emittance growth in the high intensity PSR beam. Although the artificial lattice represents an overoptimistic scenario for a stop band correction in the PSR ring, the results of the simulation provide motivation for the development of such a scheme. A real stop band correction in the PSR ring needs only to reduce or eliminate the $n=4$ harmonic associated with the stop band. The successful reduction of the driving harmonic could result in an increase of the PSR operating intensity.

Although the influence of the stop band on the emittance growth in the PSR machine has been examined, the mechanism for emittance growth, i.e., the detailed mechanics of single particles being driven to higher emittances, is not yet clear. Some discussion is dedicated to this in Ref. [11], but the topic requires further investigation.

\section{ACKNOWLEDGMENTS}

We are very grateful to the LANSCE support team for setting up and executing experiments, and to $\mathrm{T}$. Spickermann for hunting down data files and clarifying parameters. We would also like to thank V. Danilov and S.Y. Lee for their many useful insights. This work is supported by SNS through UT-Battelle, LLC, under Contract No. DE-AC05-00OR22725 for the U.S. DOE, by Indiana University through a DOE grant, DE-FG0292ER40747, and by Los Alamos National Laboratory under Contract No. W-7405-ENG-36. The Los Alamos National Laboratory is operated by the University of California for the U.S. DOE. The SNS is a partnership of six national laboratories: Argonne, Brookhaven, Jefferson, Lawrence Berkeley, Los Alamos, and Oak Ridge.

[1] I. M. Kapchinskij and V.V. Vladimirskij, in Proceedings of the 9th International Conference on High Energy Accelerators, edited by L. Kowarski (CERN, Geneva, 1959), p. 274.

[2] F. J. Sacherer, Ph.D. thesis, University of California, Berkeley, 1968.

[3] S. Machida, Nucl. Instrum. Methods Phys. Res., Sect. A 309, 43 (1991).

[4] J. Holmes, A. Aleksandrov, J. D. Galambos, F. Merrill, and R. Macek, and D. Olsen, in Proceedings of the 2000 European Particle Accelerator Conference, Vienna (CERN, Geneva, 2000), p. 936.

[5] A.V. Fedotov and I. Hofmann, Phys. Rev. ST Accel. Beams 5, 024202 (2002).

[6] R. Baartman, in Workshop on Space Charge Physics in High Intensity Hadron Rings, AIP Conf. Proc. No. 448 (AIP, New York, 1998), p. 56. 
[7] R. L. Gluckstern, in Proceedings of the Linear Accelerator Conference (LINAC70), Fermilab, edited by M. R. Tracy (Fermilab, Batavia, IL, 1970), p. 811.

[8] I. Hofmann, Phys. Rev. E 57, 4713 (1998).

[9] A. Uesugi, S. Machida, and Yoshiharu Mori, Phys. Rev. ST Accel. Beams 5, 044201 (2002).

[10] George Lawrence, in Proceedings of the 1987 IEEE Particle Accelerator Conference, Washington, DC (IEEE, Piscataway, NJ, 1987), p. 825; D. Fitzgerald, A. Browman, R. Garnett, D. Johnson, K. Jones, R. Kustom, R. Macek, F. Merrill, C. Pillai, M. Plum, and O. Sander, in Proceedings of the 1999 Particle Accelerator Conference, New York (IEEE, Piscataway, NJ, 1999), p. 518.

[11] S. Cousineau, S. Y. Lee, J. A. Holmes, V. Danilov, and A. Fedotov, Phys. Rev. ST Accel. Beams 6, 034205 (2003).

[12] J. Galambos, S. Danilov, J. Holmes, D. Jeon, F. Neri, D. K. Olsen, and M. Plum, Phys. Rev. ST Accel. Beams 3, 034201 (2000).

[13] J. Galambos, J. Holmes, A. Luccio, D. Olsen, and J. Beebe-Wang, ORBIT Users Manual, http://www. sns.gov//APGroup/Codes/Codes.htm

[14] J. Holmes, J. Galambos, D. Jeon, D. Olsen, and J. Cobb, in Proceedings of the ICAP Conference, Monterey, 1998 (SLAC, Stanford, CA, 1998), http://www.slac. stanford.edu/econf/C980914.
[15] J. Holmes, S. Cousineau, S. Danilov, S. Henderson, A. Shishlo, A. Fedotov, and S. Y. Lee, in Proceedings of the 2003 Particle Accelerator Conference, Portland (IEEE, Piscataway, NJ, 2003).

[16] J. MacLachlan, Fermilab Report No. FN-446, 1987.

[17] A. Riabko, M. Ellison, X. Kang, S. Y. Lee, D. Li, J. Y. Liu, X. Pei, and L. Wang, Phys. Rev. E 51, 3529 (1995); S. Y. Lee and A. Riabko, Phys. Rev. E 51, 1609 (1995); Chin. J. Phys. 35, 387 (1997).

[18] J. Holmes, J. Galambos, D. Olsen, and S. Y. Lee, in Workshop on Space Charge Physics in High Intensity Hadron RingsTitle (Ref. [6]), p. 254.

[19] F. Jones, Users's Guide to ACCSIM, TRIUMF Design Notes No. TRI-DN-90-17, 1990, http://www.triumf.ca/ compserv/accsim.html

[20] F. Neri, in Proceedings of the Workshop on Space Charge Physics in High Intensity Hadron Rings, Shelter Island, NY, 1998 (AIP, New York, 1998), p. 171.

[21] M. Plum, D. Brown, A. Browman, and R. Macek, in Proceedings of the 1995 IEEE Particle Accelerator Conference, Dallas (IEEE, Piscataway, NJ, 1995), p. 2554.

[22] D. Nueffer, D. Fitzgerald, T. Hardek, R. Hutson, R. Macek, M. Plum, and H. Thiessen, in Proceedings of the 1991 Particle Accelerator Conference (IEEE, Piscataway, NJ, 1991), p. 1893. 